\title{
Tunnel Operator Training with a Conversational Agent-Assistant
}

\author{
Eric Buiël, Jan Lubbers, Willem van Doesburg, and Tijmen Muller \\ TNO Defense, Security and Safety, P.O. Box 23, 3769 ZG Soesterberg, The Netherlands \\ \{eric.buiel, jan.lubbers, willem.vandoesburg, tijmen.muller\} atno.nl
}

\begin{abstract}
A tunnel operator monitors and regulates the flow of traffic inside a tunnel. Tunnel operators need to train in a simulator regularly in order to maintain proficiency in handling incident situations. During quiet working hours, the operator has enough time for training. But generally at that time no instructor or colleague operators are present to provide instruction, advises, and feedback. As a solution, we have designed an automated training system. The system employs a conversational agent which supports the operator's situation assessment tasks. The agent exhibits peer behavior which is unobtrusively directed by didactic strategies. In this paper we present the design, development and application of the agent.
\end{abstract}

Keywords: Agent-Based Modeling and Training, Cognitive Modeling, Constructive Learning, Intelligent Virtual Agent.

\section{Introduction}

The task of a tunnel operator is to monitor and regulate the flow of traffic inside a tunnel. Surveillance cameras are mounted inside the tunnel. Images from the cameras are displayed on monitors inside the control room. In case of a large-scale incident, the operator takes initial safety measures (e.g. closing traffic lanes) and stays in charge; at least until the principle fire-department officer arrives on the scene.

Tunnel operators need to train regularly in order to maintain proficiency in developing situation awareness and handling the events occurring in the stressful initial minutes of a crisis situation. We have developed a training simulator that enables a tunnel operator to train himself in incident management. The simulator presents an incident-situation to the operator, which develops in real-time. The operator has to make situation assessments and must take appropriate safety measures.

Since incidents occur infrequently, the tunnel operator's workload is unevenly distributed over time. During quiet working hours, the operator has enough time for simulator training. But generally at that time no instructor is present to provide didactic support (e.g. providing instructions and feedback). No other operators are present to provide colleague advises either. Therefore, educational support systems need to assist the operator during the training. Intelligent virtual agent technology enables the creation of virtual characters that conduct a true dialogue with humans [1] [2]. Intelligent virtual agent technology combines a realistic real-time three-dimensional 
visualization of a human, with the cognitive modeling of 'what is on the human's mind', and means to communicate in spoken and written word. The application of agent technology enables us to create virtual agents that provide didactic support to the tunnel operator, for example virtual instructors and virtual co-learners. Yet, not much is known about the most effective way of applying these virtual agents for operator training.

\section{Research Goals and Approach}

In this paper we present the design, development and application of an educational agent for the training of a tunnel operator. The main research question of this project was: What are the necessary agent functionalities for virtual agents that accompany a tunnel operator during task training in a tunnel training simulator? Here, we considered knowledge requirements (i.e. which domain knowledge, didactic knowledge, and student knowledge has to be available for the agent?) as well as behavioral requirements (i.e. what is the most effective content, form and timing of the agent's interventions and instructions?). We first performed a literature study on agent-based training applications. Based on the results of this study, we composed a generic framework that describes the cooperation between a human supervisor and an educational agent jointly training a supervisory task, like the tunnel operator task. Based on this framework, we implemented a prototype educational agent in our tunnel training simulator. In future projects, this prototype can be used to refine the agent design guidelines by means of training experiments.

This paper provides an overview of the project results. $\S 3$ presents an overview of generic design guidelines for educational agents obtained from literature. $\$ 4$ through $\$ 7$ describe the agent-student cooperation framework and the prototype educational agent. $\S 8$ concludes with a summary and the focus of future training experiments.

\section{Educational Background}

In 2000, Johnson [3] recognized that animated pedagogical agents were in the early stages of development, but that they would have a significant impact on education and training in the near future [4] [5]. Engaging, expressive pedagogical agents can provide feedback and advice that have a strong motivating effect on trainees, and may even encourage and empathize with these trainees. Moreno et al. [6] conducted a study investigating the effectiveness of an animated pedagogical agent on children's learning. One group of trainees learned with on-screen instruction and the help of a pedagogical agent and another group learned just by reading on-screen instructions. Across two separate experiments, the agent group had a $24-48 \%$ higher success rate than the non-agent group. For children, subtle praising for effort proves to provide good result; better than praising for accomplishment [7].

Another effect that can be expected from a life-like agent is that the trainee will build up a relationship with an agent and might become committed to respond to the agent in a positive way. According to Kidd and Breazeal [8], the most important factors for trying to create and maintain a helpful, long-term human-agent relationship 
are engagement, trust and motivation. The agent used relational strategies such as social dialogue, empathy dialogue, meta-relational communication, humor, continuity behaviors, and forms of address and politeness strategies [9]. Personality is fundamental to social relationships. People automatically perceive a personality in social agents even when no personality is intended [10]. This has led to the "computer as a social actor" paradigm put forward by Reeves and Nass [11]. In this paradigm all computer mediated interaction should take human social assumptions into account. Therefore, explicitly adding a personality can direct the social interaction into the desired direction.

The classical student-teacher learning method implies a hierarchical relationship between student and instructor instead of a social relationship. The instructor chooses the exercises, directs the learner, evaluates task performance, and exactly tells the learner how to act in order to improve performance. This classical method does not match sufficiently with the "computer as a social actor" paradigm. Modern, selfdirected, constructive learning methods [12] [13] provide a better match. Here the trainee is fully responsible for his own task proficiency. The trainee is encouraged to improve his proficiency, preferably by experimenting and by cooperating with colleague trainees. Trainees discuss their task strategies together, and think about alternative strategies that might improve task performance. As a consequence, social interaction between trainees is crucial to the success of these learning methods.

In order to implement the above-mentioned social, constructive learning approach in an agent-based learning environment, like the tunnel training simulator, we need an educational agent acting like a co-learner. This companion agent encourages the trainee to discuss his thoughts and actions and stimulates discoveries on how to reach the learning goals. The agent has the same expertise as the operator does. This means that the agent can provide good suggestions, but might also make mistakes. The trainee does not have to follow the agent's advice. The agent just needs to make him think over certain situations that occur during the scenario execution.

In our tunnel training simulator, we implemented a prototype companion agent (§4) that supports the trainee in situation assessment tasks (§5). The design framework of the agent is described in $\$ 6$. $\$ 7$ exemplifies typical agent interventions.

\section{Demonstration Setup}

Figure 1 shows the tunnel training simulator with companion agent. The tunnel training simulator implements a virtual model of the tunnel control room. Instead of operating on a real tunnel control system, commands are sent to a virtual tunnel simulator and sensor signals (surveillance camera pictures, alarm messaging from automated surveillance systems) are received in return. The trainee and companion agent interact socially while executing their joint task. In Figure 1, the companion agent is indicated by (1), the virtual tunnel control system is indicated by (2), and the virtual tunnel monitor is indicated by (3). In this setup, the operator can switch easily between operating the control system, monitoring the tunnel, and interacting with his co-learner.

The companion agent can interact with the trainee through verbal communication, with either written or spoken dialogue. The former is implemented by a text based input module, the latter by a voice recognition module. The agent is embodied 


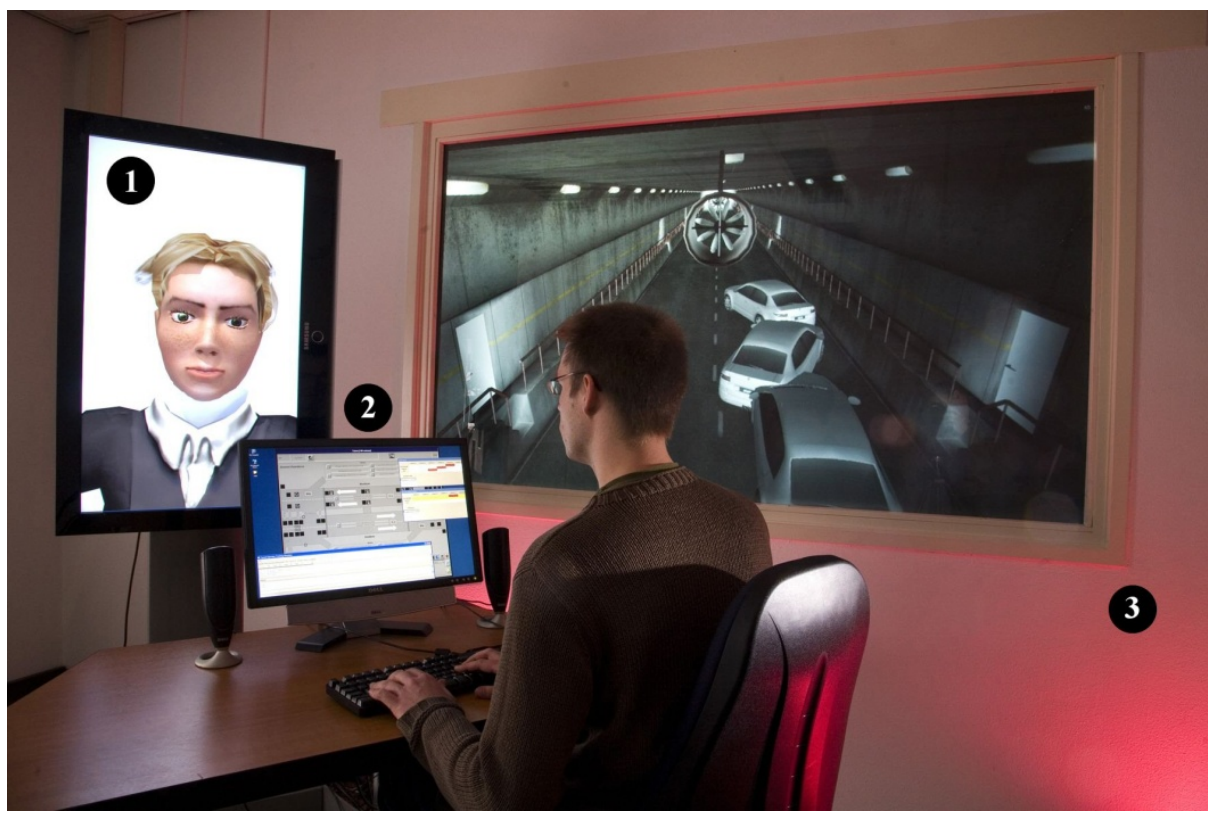

Fig. 1. Tunnel training simulator with a companion agent

through an animated 3D character model, and a speech synthesis module transforms written dialogue to speech, which is fed into a lip-synchronization module. The behavior of the agent is discussed in $\S 6$.

\section{The Task of the Tunnel Operator}

Tunnel incidents are categorized and ranked by their impact on tunnel safety. For each incident type, a different incident procedure exists. The tunnel operator needs to execute this procedure in order to return to a safe situation. E.g. for a simple car crash without injuries and fire, he needs to close the traffic lane and call in the tow service. For a large-scale fire incident, he needs to push an alarm button that activates automatic safety measures (e.g. activating route signing of emergency exits). Table 1 shows the incident types of the tunnel training simulator.

Table 1. Incident classifications in the tunnel training simulator

\begin{tabular}{|c|l|c|c|c|}
\hline \multirow{2}{*}{$\begin{array}{c}\text { Incident } \\
\text { type }\end{array}$} & \multicolumn{2}{|c|}{$\begin{array}{c}\text { Incident } \\
\text { name }\end{array}$} & \multicolumn{3}{|c|}{\begin{tabular}{c} 
Events occurring inside the tunnel \\
\cline { 3 - 5 }
\end{tabular}} & & Damage & Injuries & Fire \\
\hline 0 & No incident & No & No & No \\
\hline 1 & Incident with material damage & Yes & No & No \\
\hline 2 & Incident with injuries & & Yes & No \\
\hline 3 & Fire incident & & & Yes \\
\hline
\end{tabular}


The task of the tunnel operator is largely a situation assessment task. In order to perform well, the tunnel operator must have good situation awareness (SA). Endsley [14] defines SA as: "the perception of the elements in the environment within a volume of time and space, the comprehension of their meaning, and the projection of their status in the near future". Translated to the specific tunnel operator task, the operator needs to do observations (phase 1 of SA, perceptions) and use these to classify the current situation in the form of a diagnosis (phase 2 of SA, comprehension). Based on the diagnosis, the operator decides on a course of action (the third phase of SA, projection).

Learning objectives. From a situation awareness perspective, the learning goals of tunnel operator training are threefold:

1. The trainee is able to find the information that is necessary to make a situation assessment (the observation objective, related to the first phase of SA);

2. The trainee is able to classify an incident situation by combining individual observations into a correct situation assessment (the diagnosis objective, related to the second phase of SA);

3. The trainee has developed "critical thinking" skills [15]. From time to time, he needs to reassess the situation, and decide whether his current situation classification is still correct (the critical thinking objective).

In the current simulator, the learner does not have to decide on the course of action; in other words, projection is not a learning objective in this implementation.

Error types. The trainee may fail to perform correct diagnosis formation due to a number of reasons. We categorize five error types in total, divided over the learning objectives mentioned above. For the observation objective these errors are:

1. the unseen error: the trainee has failed to notice something;

2. the hallucination error: the trainee reports something that does not take place.

For the diagnosis objective, there are two typical errors:

3. the classification error: the trainee has not applied the incident classification rules correctly;

4. the omission error: the trainee has not shared an observation with his peer.

Finally, there is one typical error within the context of the critical thinking objective:

5. the critical attitude error: the trainee does not reassess the situation frequently, or holds on to an obsolete diagnosis too long.

\section{The Cooperation between Learner and Agent}

For effective constructive learning, it is vital that the trainee is able to speak freely with his co-learner, without feeling judged. This implies the absence of an authoritarian relationship. Therefore, the agent must act as a true companion to the trainee. As such, the agent is required to exhibit peer behavior to create a safe and trustworthy 
atmosphere. At the same time, the agent should stimulate conversation with the trainee in order to encourage him to explain his reasoning and to enable him to do new discoveries and construct new knowledge. In order to achieve this, the agent is required to guide the didactic process. Didactic guiding must occur only unobtrusively and must remain unnoticed by the trainee.

Figure 2 illustrates how these two principles (peer behavior and didactic guiding) are incorporated in the design of the agent. The agent is divided in two separate layers: the didactic layer and the peer behavior layer. The top layer (didactic module) contains domain-independent didactic knowledge. In the bottom layer (peer behavior module), the behavior of the agent is modeled to represent the role of a companion to the trainee. On this layer the specific domain knowledge is modeled. This design enables easy reuse of the agent for training other supervisory tasks. Only the specific domain knowledge in the peer behavior module needs to be remodeled.

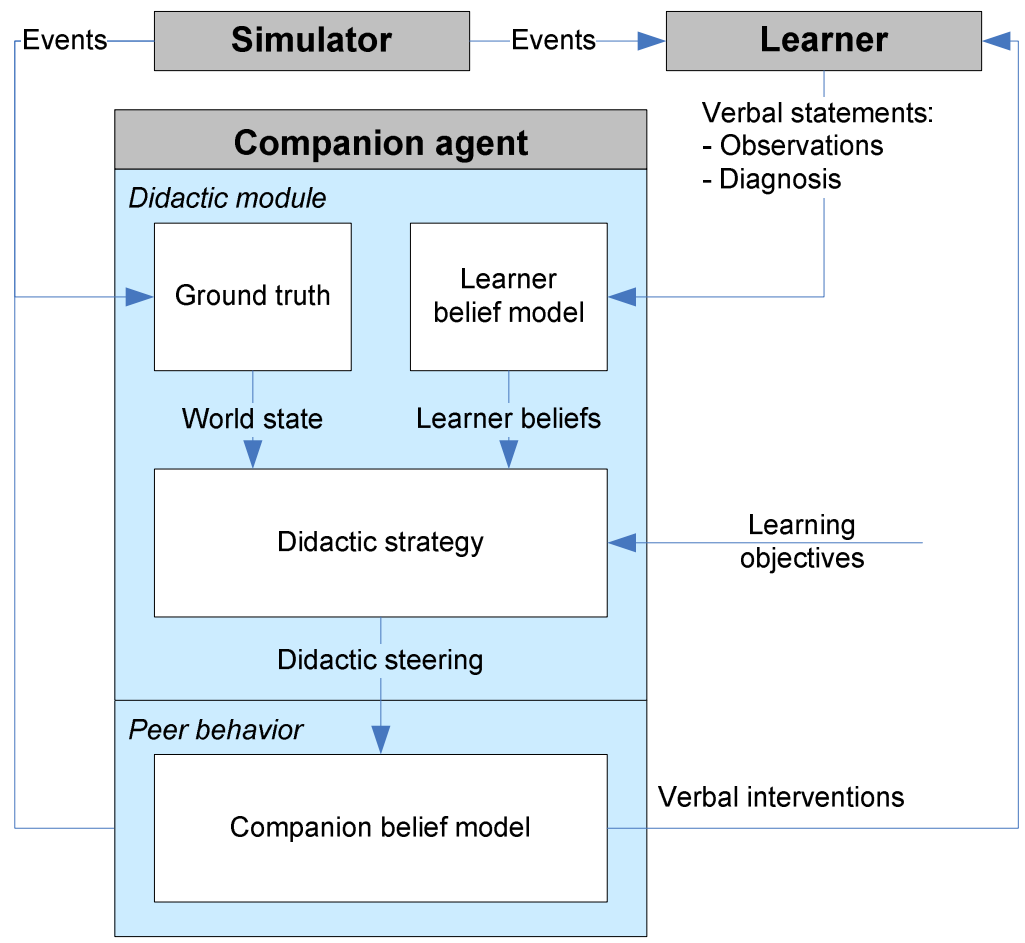

Fig. 2. Companion agent design framework

The agent's peer behavior is executed by the peer behavior module and directed by the didactic module. The didactic module has a complete view on the world, whereas the peer behavior module and the trainee only have partial (and possibly incorrect) views on the world. These views are based upon events sent by the simulator.

The trainee can share his beliefs with the agent verbally by expressing statements. Each statement can either regard a new observation (e.g. "I see car damage on camera 5.") or a situation diagnosis (e.g. "There is a fire incident.”). These statements 
are semantically processed into beliefs, which are added to the learner belief model. The didactic module compares the learner's beliefs to the ground truth in order to recognize and classify possible errors (see $\$ 5$ ). The result of this classification is used to direct the peer behavior of the agent. The didactic module determines if the trainee's observations are correct, sufficient and necessary to support his diagnosis. Depending on the classification, different didactic strategies can be applied. The didactic module employs several dialogue models to encourage the trainee to explain his diagnosis, including asking the trainee for supportive evidence, encouraging the trainee to state his observations, and intervening (e.g. deny an observation, suggest a review of the observation, or propose a different action).

\section{Typical Agent Interventions}

For each learning objective, this section describes the most typical agent interventions.

The observation objective. If the trainee's latest verbal statement indicates a new observation, the agent will respond following the flowchart in Figure 3 . If the observation is incorrect, the provided verbal intervention depends on the type of observation error. For example, when the trainee incorrectly observes that injuries are present (hallucination error), the agent will focus on more detailed observation, and respond "I'll go check the number of injuries." Another example is when the trainee incorrectly observes that injuries are not present (unseen error), the agent will focus on typical incident characteristics, and respond "There is a chance that people are hurt." For each error type, multiple response phrases are available, such that the agent can select a different response phrase when the trainee holds on to an incorrect observation.

If the observation is correct, the agent will stimulate the trainee to investigate if this observation changes the diagnosis.

The diagnosis objective. If the trainee's latest verbal statement indicates a new diagnosis, the agent will first ask the trainee to explain his diagnosis by calling out the observations that support the diagnosis one at a time. Subsequently, the agent will respond following the flowchart in Figure 4.

If (some of) the supporting observations are incorrect, or if the trainee did not mention crucial information in relation to the provided diagnosis, the agent will stimulate the trainee to reconsider his observations. The agent categorizes the incorrect or missing observations as hallucination errors, unseen cue errors, and omission errors, and selects a single error from the observation set. If the selected error is a hallucination error or an unseen error, the agent uses verbal responses similar to Figure 3 , in order to encourage the trainee to reconsider his diagnosis. If the selected error is an omission error the agent tells the trainee his own beliefs regarding the specific subject. For example, suppose the trainee correctly diagnosed the incident situation as an incident with injuries (incident type 2 in Table 1). When the trainee did not mention the absence of fire in his diagnosis explanation, the agent responds "I think there is a fire." This statement is incorrect, and probably the trainee will correct the agent. But at the 


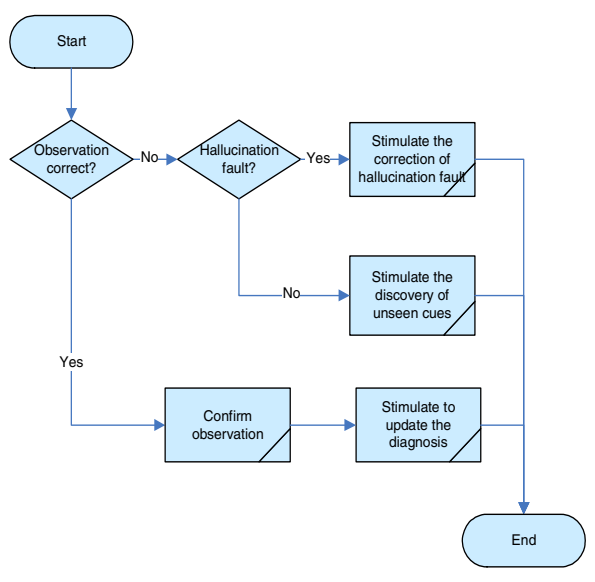

Fig. 3. Handling new observations

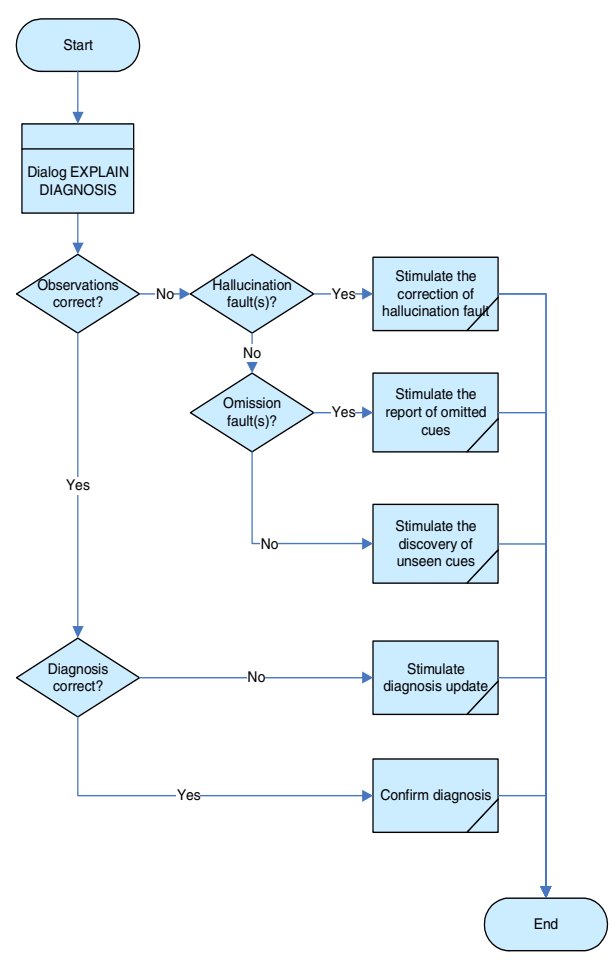

Fig. 4. Handling new diagnoses

same time, it will probably make him think over the relation between the presence/absence of fire and the diagnosis of a type 2 incident.

If the trainee's observation set is correct and complete, but his diagnosis is incorrect (classification error), the agent will stimulate the operator to reconsider his classification by providing his own diagnosis belief. Once more, this belief may even be incorrect.

The critical thinking objective. If the trainee's diagnosis belief does not change quickly after an event in the simulated tunnel (i.e. a change of the ground truth), the agent concludes the trainee holds on to an obsolete diagnosis. Then, the agent provides unobtrusive hints in order to stimulate the trainee to suspend his current activities and start a critical review of his current diagnosis belief. This is likely to occur when shortly after a first incident, a second incident occurs. If the second incident remains unnoticed by the trainee, the agent will first ask "What is going on?" If the trainee holds on to his initial diagnosis the agent will ask "Which procedure did you follow?" Subsequently he will ask "When did it happen?" The last-mentioned question causes the trainee to place the earlier observed events in a time frame, and encourages the trainee to investigate whether current events are in line with the events observed earlier. 


\section{Conclusions and Future Work}

In order to apply virtual agents for training, the social relationship between the agent and the trainee has to be taken into account. The agent must consciously balance between motivating and guiding the learner. On the one hand, the agent must prominently act as a true companion to the trainee, and exhibit peer behavior to create a safe and trustworthy atmosphere. On the other hand, the agent must unobtrusively guide the didactic process: the agent must encourage the trainee to verbally explain his reasoning in order to enable him to do new discoveries and construct new knowledge. Didactic guiding must remain unnoticed by the trainee.

Our agent-learner cooperation framework (\$6) enables the creation of social educational agents supporting a human supervisor when training a supervisory task. The implemented companion agent prototype for tunnel operator training shows the capabilities at hand within this framework. Future experiments have to show if training with this agent indeed results in improved situation awareness and solid incident assessments. These experiments also have to provide the most effective content, form and timing of the agent's interventions and instructions.

\section{Acknowledgments}

The research reported here is part of the Interactive Collaborative Information Systems (ICIS) project, supported by the Dutch Ministry of Economic Affairs, grant nr. BSIK03024.

\section{References}

1. Rickel, J., Marcella, S., Gratch, J., Hill, R., Traum, D.R., Swartout, W.R.: Toward a New Generation of Virtual Humans for Interactive Experiences. IEEE Intelligent Systems 17(4), 32-38 (2002)

2. Traum, D.R., Rickel, J.: Embodied agents for multi-party dialogue in immersive virtual worlds. In: Proceedings of the first International Joint conference on Autonomous Agents and Multiagent systems, pp. 766-773 (2002)

3. Johnson, W.L., Rickel, J.W., Lester, J.C.: Animated pedagogical agents: Face-to-face interaction in interactive learning environments. International Journal of Artificial Intelligence in Education 11, 47-78 (2000)

4. Atkinson, R.K.: Optimizing learning from examples using animated pedagogical agents. Journal of Educational Psychology 94, 416-427 (2002)

5. Clark, R.C., Mayer, R.E.: e-Learning and the Science of Instruction: Proven Guidelines for Consumers and Designers of Multimedia Learning. John Wiley \& Sons. Inc., San Francisco (2003)

6. Moreno, R., Mayer, R.E., Spires, H., Lester, J.: The case for social agency in computerbased teaching: Do trainees learn more deeply when they interact with animated pedagogical agents? Cognition and Instruction 19, 177-214 (2001)

7. Mueller, C.M., Dweck, C.S.: Praise for intelligence can undermine children's motivation and performance. Journal of personality and social psychology 75, 33-52 (1998) 
8. Kidd, C.D., Breazeal, C.: Sociable robot systems for real-world problems. In: IEEE International Workshop on Robot and Human Interactive Communication, pp. 353-358 (2005)

9. Bickmore, T.W., Picard, R.W.: Establishing and maintaining long-term human-computer relationships. ACM Transactions on Computer-Human Interaction (TOCHI) 12, 293-327 (2005)

10. Dryer, D.C.: Getting personal with computers: How to design personalities for agents. Applied Artificial Intelligence 13, 273-295 (1999)

11. Reeves, B., Nass, C.: The Media Equation: How People Treat Computers, Television, and New Media Like Real People and Places. Cambridge University Press, Cambridge (1996)

12. van der Hulst, A., Muller, T.J., Besselink, S., Coetsier, D., Roos, C.L.: Bloody Serious Gaming - Experiences with Job Oriented Training. In: Interservice/Industry Training, Simulation, and Education Conference (I/ITSEC), Orlando, FL (2008)

13. Stehouwer, M., Stricker, J., van Gemeren, W.: Training Design for Professional Development. In: Interservice/Industry Training, Simulation, and Education Conference (I/ITSEC), Orlando, FL, p. 12 (2006)

14. Endsley, M.R.: Toward a theory of situation awareness in dynamic systems. Human Factors 37(1), 32-64 (2005)

15. van den Bosch, K., Helsdingen, A.S.: Critical Thinking in Tactical Decision Games Training. In: Cook, M., Noyes, J., Masakowski, Y. (eds.) Decision Making in Complex Environments, pp. 213-222. Ashgate, Aldershot (2007) 